\title{
Different Nasolabial flaps in nasal reconstruction
}

A.S.Ezzat, A.M.Abdelmofeed, M.A.Mohamed and A.F.Ebrahim

General Surgery, Dept., Faculty of Medicine, Benha Univ., Benha, Egypt

E-Mail: Amira fathy286@gmail.com

\begin{abstract}
Nasal reproduction is a difficult plastic medical procedure, it is the most unmistakable and stylish component of the face. Point of work is assessment, result and complexity of the nasolabial folds in nose recreation. Strategies: This examination was done in Benha University Hospital and Benha Health Insurance Hospital on thirty patients with various nasal imperfections. Subsequent to assessing the site, size, and profundity of the imperfections, recreation was finished by nasolabial folds. The folds were planned by the deliberate nasal deformities. Results and end: The mean age of the examination members was 57 years, with a standard deviation of 10 years. As to, $76.7 \%$ were guys, while $23.3 \%$ were females. The nasolabial fold is a simple, snappy and successful methodology for treatment of more modest and average estimated imperfections of the midfacial region. This method permits collecting a wide and rich vascularized fold along the entire length of the nasolabial overlap. In blend with the wide circular segment of pivot, the fold could arrive at practically any site of centrofacial territory. The closeness to the beneficiary deformities, the best shading match and the acceptable form made from the generally bare territories used from the nasolabial overlap are the other significant benefits of the method.
\end{abstract}

Keywords: Nasolabial, Flaps, Reconstruction.

\section{Introduction}

Nasal reproduction is a difficult plastic medical procedure, it is the most unmistakable and stylish component of the face [1].

Nose assumes a part on eye to eye correspondence by being in the focal point of face. The focal area and projection makes its general stylish significance and contributesin continuous injury. Nasal deformities might be brought about by inherent contortion, contamination, injury, and neoplasm [2].

In view of the little nearby tissue and restricted skin versatility, reproduction of the nasal deformity actually makes trouble for the plastic specialist. The standard of the reproduction ought to keep up the nearby characteristicssuch as the evenness, shape, surface, and useful uprightness byusing a less horrible activity [2].

Cheek tissue has the very tone and surface to that of nose, and its vicinity accommodates simpler transpositionof the fold. Likewise, negligible benefactor site distortion. The nasolabial fold is ideal for nose recreation. Easyharboring and capacity work under nearby sedation with relative negligible scar makes it the most utilized folds in reproduction of nasal imperfections [3].

The nasolabial fold has been usedfor facial recreation since the nineteenth century. The previously recorded depiction traces all the way back to an Indian Surgeon, Sushruta, in 600 BCE. Effectively reap, solid blood supply, and capacity of the actual fold for various applications [3].

Nasolabial folds have fluctuates alterations for accomplishing the best outcome [2]. Most alterations for the nasolabial folds are V-Y ax headway, rendering, subcutaneous pedicled, two phase insertion, and propeller types. .Each sort of nasolabial fold has its benefits and disservices and each partof the nose has interesting highlights which needs unique attentionin performing recreation [4].

Point of work is assessment, result and difficulty of the nasolabial folds in nose remaking.

\section{Patients and methods}

This imminent, non-randomized clinical examination included thirty patients gave clear nasal imperfections conceded to Benha medical coverage Hospital and Benha college Hospital in the time frame from December 2019 to December 2020. After an endorsement from the Research Ethics Committee in Benha Faculty of medication and completely educated composed assent from all patients as respect surgery in this exploration has been gotten from all patients and outright privacy as respect the patients' names and addresses was given exceptional consideration and consideration., shooting and follow up period.

\subsection{Inclusion criteria}

- Age: any age

- Gender: males \& females.

- cooperative, fit for surgery

\subsection{Exclusion criteria:-}

- Patient with large defects.

- Smoking.

- Patients with post burn nasal defects.

- Severe chronic illness as (chronic renal failure, chronic liver disease, etc....).

- Mentally or psychologically disordered patients.

- Presence of active infection

All Patients were subjected to a detailed thorough history taking, clinical examination, investigations, operative procedure and postoperative assessment and follow up.

Informed consent was obtained for all patients which was clearly documented

Nasal lesions were specified regarding to the site, size, depth of defects and the previous medical or surgical treatment.

\section{Steps:}

- All patients were operated on by the same surgeon.

- Oral broad spectrum antibiotic was started one day before surgery. 
- preoperative marking

- Intra-operative analysis of nasal defects :

- Anatomical site of the defect in nose, involvement of aesthetic unit.

- Size of the defect.

- Depth of the defect (Skin only or skin and cartilage or full thickness defect.

- Involving of lining mucosa.

\subsection{Anaesthesia}

- General anaesthesia or local anaesthesia with intra venous sedation according to patient general condition.
- Supine position, head at $20 \_30$ degrees with the head in the midline or turned to the opposite side.

- Eye protection by sterile tapes.

\subsection{Tumor excision}

- The tumors were resected with 4-5 mm lateral safety margins in basal cell carcinoma, $10 \mathrm{~mm}$ in squamous cell carcinoma.

- Specimen was labeled for histopathological examination.

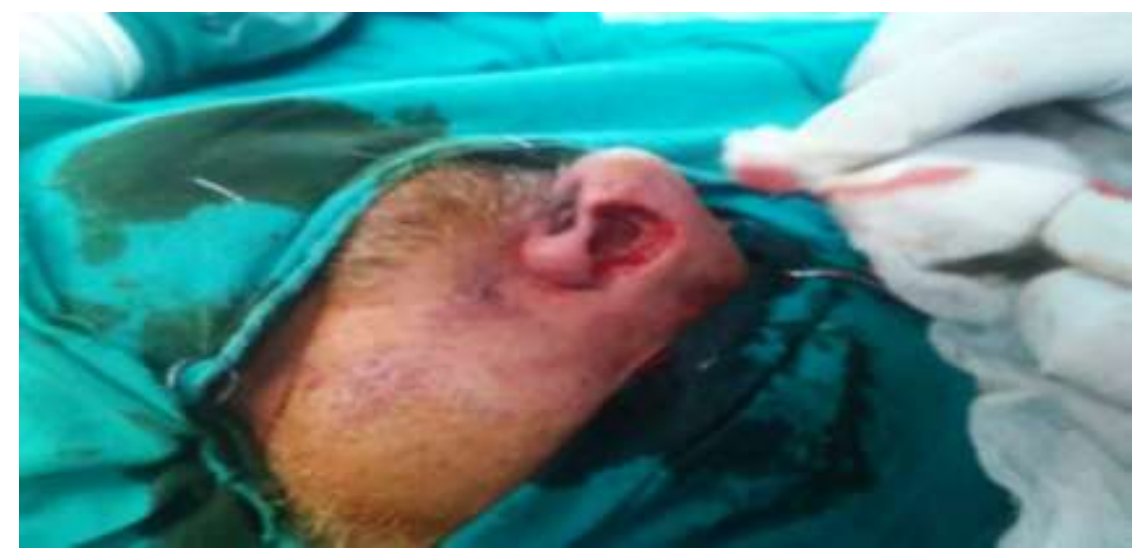

Fig (1) Nasal defect after tumor excision .

\subsection{Flap Design}

- A foil pattern template of the defect was used for the flap design.

- Injection with 1:200,000 epinephrine for haemostatic control and to aid in flap dissection.
- The flaps were designed according to the measured nasal defects, either superiorly based or inferiorly based according to the pattern of the nasal defects.

- In some cases especially with the alar defects, the nasal tissue (skin and subcutaneous tissue) between the nasal defects and the medial border of the flaps are removed in one stage nasal reconstruction.

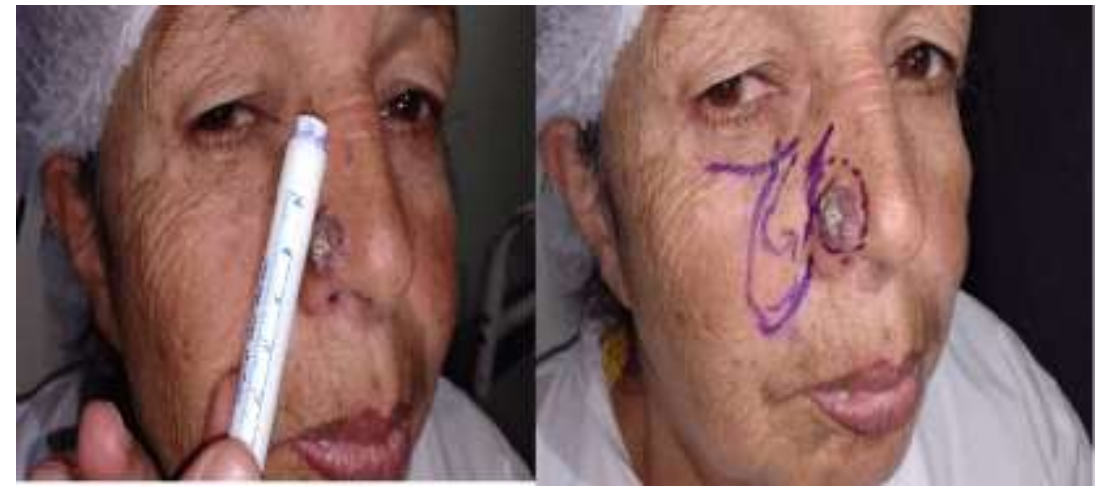

Fig (2)preoperative marking of superiorly based nasolabial flap.

\subsection{Surgical technique}

The nasolabial fold was marked preoperatively on all patients. A template was positioned immediately adjacent to the nasolabial fold ensuring an appropriate arc of rotation from the donor to the recipient site. A distal dog ear excision was outlined.
Care was taken to ensure that the lower portion of the nasolabial fold was included in the lower portion of the incision as on closure of the donor site, the existing nasolabial fold crease becomes the inferior margin of the incision. 
The fold was raised forcefully to the level of the mid cheek and took apart gruffly with care to the base of the flap. The fold was progressed onto the nose; conveying the distal expansion to cover the deformity. Hemostasis was finished with electrocautery.

The fold was then molded and formed to be more modest than the imperfection this permits the fold to be inset under a level of strain. This is the main advance when utilizing the nasolabial fold for nasal tip recreation.

The fold was fixed with stitches to the beneficiary site taking consideration to guarantee wound edge conclusion with eversion. To limit unmistakable giver site scarring, the fold configuration was never reached out over the level of the alar lobule.

The fold contributor locales were shut cautiously with numerous layers of profound dermal stitch followed by nylon skin stitches. Dressing was finished.

\subsection{Superiorly based flap}

The fold planned so the second rate tip of the fold limits to a point. This plan will take into consideration the conclusion of the contributor site with minimal measure of sabotaging and extraction of canine ear. The fold is raised from the distal tip towards the base by first making a cut profound to the dermis along the stamped width of the fold.

The fold is raised in a plane shallow to the muscles. Care ought to be taken to recognize and dodge injury to the opposite parts of the facial course as they enter the muscle on their approach to perfuse the overlying skin.The encompassing territory is subverted to improve the pivot of the fold without causing twisting of the tissues around the base of the fold.

In situations where the fold is to be utilized along the nasal sidewalls, the fold is turned to safeguard the span is satisfactory and without strain. The fold is formed to the deformity and inset by putting one to two profound stitches along the base to reproduce the furrow.

\subsection{Statistical techniques}

Information the board and measurable examination were finished utilizing SPSS vs.25. (IBM, Armonk, New York, United States).Numerical information were summed up as means and standard deviations. All out information were summed up as numbers and rates.

\section{Results}

This study was done in Benha University Hospital and Benha Health Insurance Hospital on thirty patients with different nasal defects. After evaluating the site, size, and depth of the defects, reconstruction was done by nasolabial flaps. The flaps were designed according to the measured nasal defects. The mean age of the study participants was 57 years, with a standard deviation of 10 years. Regarding gender, $76.7 \%$ were males, while $23.3 \%$ were females. Table (1).

Table (1) Demographic characteristics of the studied patients.

\begin{tabular}{llc}
\hline \multicolumn{2}{c}{ Demographics } \\
\hline Age (years) & Mean $\mathbf{\pm S D}$ & $57 \pm 10$ \\
& Males' n (\%) & $23(76.7)$ \\
Gender & Females' n (\%) & $7(23.3)$ \\
\hline
\end{tabular}

The most frequent lesion was BCC (73.3\%), followed by SCC (16.7\%). Traumatic lesions were present in only $10.0 \%$ of patients, fig (3).

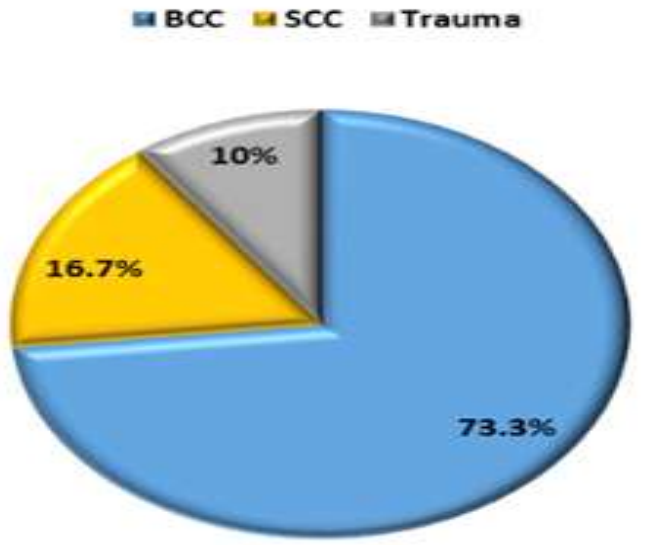

Fig (3)Types of lesions in the studied patients.

The most frequent lesion site was Ala $(73.3 \%)$, followed by tip (40.0\%). Sidewall lesions were present in only $16.7 \%$ of patients Fig (4). 


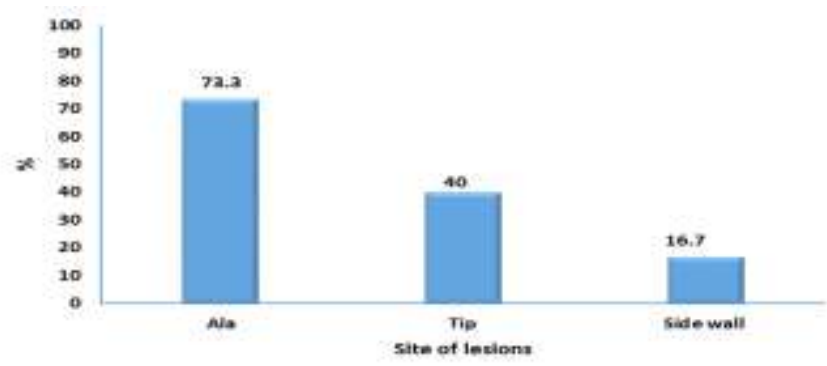

Fig (4) Site of lesions in the studied patients.

More than two-thirds of patients had lesions sized $<2$ $\mathrm{cm}$. lesions sized $>2 \mathrm{~cm}$ represented $26.7 \%$ of the studied patients.
Regarding the depth of lesions, Most of the lesions were skin only $(53.3 \%)$, while $26.7 \%$ were skin + cartilage, and $20.0 \%$ were full-thickness lesions Table (2).

Table (2) Size \& depth of lesions in the studied patients.

\begin{tabular}{lcc}
\hline & & $\mathbf{n}(\boldsymbol{\%})$ \\
\hline Size $(\mathbf{c m})$ & $\leq 2$ & $22(73.3)$ \\
& $\geq 2$ & $8(26.7)$ \\
Depth of defect & Full-thickness & $6(20.0)$ \\
& Skin only & $16(53.3)$ \\
& Skin+cartilage & $8(26.7)$ \\
\hline
\end{tabular}

Regarding donor site morbidity, only $6.7 \%$ of patients reported late morbidity in form of hypertrophic scar, while $93.3 \%$ didn't report any morbidity. Regarding recipient site morbidity, one patient reported wound dehiscence $(3.3 \%)$ as an early morbidity, late recipient site morbidity one patient reported alar distortion $(3.3 \%)$, one patient reported partial distal flap necrosis $(3.3 \%)$, and one patient reported tip deformity (3.3\%), while $86.7 \%$ of patients reported no recipient site morbidity Table (3).

Table (3) Donor and recipient site morbidity in the studied patients.

\begin{tabular}{|c|c|c|}
\hline & Early n(\%) & Late $\mathbf{n}(\%)$ \\
\hline Donor site morbidity & None $28(93.7)$ & Hypertrophic scar 2 (6.7) \\
\hline \multirow[t]{5}{*}{ Recipient site morbidity } & Partial wound dehiscence 1 (3.3) & Alar distortion 1 (3.3) \\
\hline & & Partial distal flap necrosis \\
\hline & & $1(3.3)$ \\
\hline & & Tip deformity 1 (3.3) \\
\hline & & None $26(86.7)$ \\
\hline
\end{tabular}

All patients (100.0\%) reported good functional outcomes as normal breathing and no airway obstruction, Regarding aesthetic outcomes The Likert scale shown in fig (5).

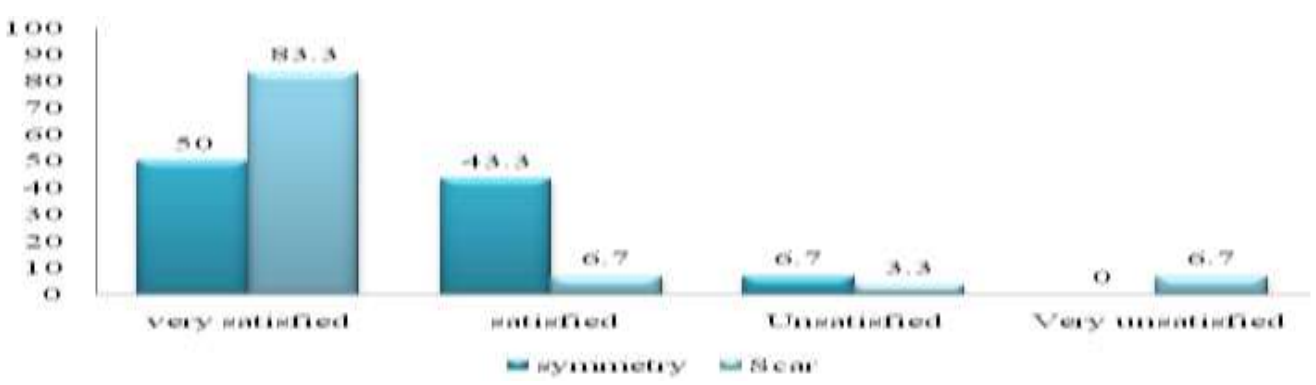

Fig (5) Aesthetic outcomes and patient satisfaction in the studied patients.

The most frequent flap-type was superiorly based flap (60.0\%), followed by inferiorly based flap (23.3\%), and freestyle perforator nasolabial island flap was the least frequent one (16.7). 


\section{Discussion}

We would say and that of Weathers the nasolabial fold is quite possibly the most generally utilized reconstructive choices for nasal imperfections [5]. The closeness of the nasolabial area, having a vigorous blood supply and excellent giver scar are the critical benefits of nasolabial folds. The perforators of rakish part of facial vein feed the nasolabial district and give many fold choices for nasal recreation. Notwithstanding, the restrictions of the fold use incorporate; the restricted fold size, width and the restricted curve of revolution. This makes it just appropriate for little and medium measured imperfections. A speculation upheld by EL-MARAKBYexperience [6].

The procedure of collecting a nasolabial fold is very functional, with a simple expectation to absorb information with negligible contributor site bleakness. This fold can be utilized singularly or respectively as superiorly, poorly or midway based pedicle fold. In the superiorly based nasolabial fold, the base of the fold is close to the ala and the pinnacle is in accordance with the oral commissure. Once in a while when an additional length is required, it very well may be reached out to the skin over the mandibular boundary. This variation of the superiorly based nasolabial fold is called expanded nasolabial fold. In the poorly based nasolabial fold the summit of the fold is 5-7 $\mathrm{mm}$ parallel to the average canthus [7].

Most of the nasolabial folds are arbitrary example, however can be intended to have a hub design blood supply from the poorly based hub nasolabial fold that is supported by facial corridor and the superiorly based converse stream nasolabial fold containing precise vein [8].

Fujiwaraused this fold as a bilobednasolabial fold for his situation report [8], while Spear and his partners [9] gave a curve to the nasolabial fold to fix ala. In our examination, we utilized it as a superiorly, poorly based or perforator based nasolabial fold.

In the current investigation, the majority of the nasolabial folds were raised neighborhood sedation, with a short technique time (26-46 minutes), an outcome that matches EL-MARAKBYexperience [6].

In our examination we experienced one patients experienced halfway fold putrefaction, one had fractional injury dehiscence and two had hypertrophic scar out of 30 cases. In an examination directed by Silistreli [10] one patient endured halfway nasolabial fold putrefaction and two had a hypertrophic scar in a sum of 10 cases. Anyway the investigation of Burgetand Hassan uncovered practically no entanglement with this fold [11].

In Javaid [12] study they revealed the result of nasolabial fold in remaking nasal alar deformities. Despite the fact that they accomplished great outcomes with the fold, alar contortion happened in $5.71 \%$ of patients and fold tip disfigurement in $2.86 \%$ patients. In our current examination these intricacies address $3.3 \%$ of the cases.

In this investigation, the shading match was acceptable on the whole instances of nasolabial fold. The benefactor site recuperated well with no useful dreariness. This is like outcomes acquired by Irfanullah [13].

In this investigation and of Bilal [14] no venous clog noticed post operatively, and this isn't equivalent to Hassan
[11] experience in which Superiorly based fold is more responsible for creating pin padding and edema than poorly based in light of fold plan lymphatic and venous seepage are more at risk for blockage, and this clog is more if the patient is wearing eye glasses as it might bother fold edema.

While superiorly based is more solid if there should be an occurrence of full-thickness alar imperfection than poorly based fold. In cases with full-thickness alar deformity, reproduction were finished by superiorly based fold with no utilization of chondrocutaneous unite with a decent useful and tasteful result and this isn't tantamount to the examination the investigation by Massoud [15].

Massaoud [15] revealed a half pace of update a medical procedure to lessen the majority of the ala and to situate the alar side divider in a more average area. This reality was not noted in the current examination.

We accomplished a decent form and shading match in our patients with no significant complexities. Benefactor site scar isn't apparent, since the scar rests in the nasolabial sulcus. The activity time, short clinic stay, similarity of the tissue tone, giver site scar area, patient solace, auxiliary systems, and cost-viability are motivations to favor this strategy over other fold strategies.

\section{Conclusion}

The nasolabial fold is a simple, speedy and successful methodology for treatment of more modest and fair measured deformities of the midfacial zone. This procedure permits reaping a wide and rich vascularized fold along the entire length of the nasolabial overlay. In blend with the wide circular segment of turn, the fold could arrive at practically any site of centrofacial zone. The closeness to the beneficiary deformities, the best shading match and the palatable form made from the generally bare zones used from the nasolabial overlap are the other significant benefits of the method.

\section{References}

[1]D. J. Singh and S. P. Bartlett, "Aesthetic considerations in nasal reconstruction and the role of modified nasal subunits.," Plast. Reconstr. Surg., vol. 111, no. 2, pp. 639-648, 2003.

[2]W. F. Larrabee Jr, "Design of local skin flaps," Otolaryngol. Clin. North Am., vol. 23, pp. 899-923, 1990.

[3]R. R. CAMERON, W. D. LATHAM, and J. A. DOWLING, "Reconstructions of the nose and upper lip with nasolabial flaps," Plast. Reconstr. Surg., vol. 52, pp. $145-150,1973$.

[4]G. C. Burget and F. J. Menick, "The subunit principle in nasal reconstruction.," Plast. Reconstr. Surg., vol. 76, pp. 239-247, 1985.

[5]E. A. Chu and P. J. Byrne, "Local flaps I: bilobed, rhombic, and cervicofacial," Facial Plast. Surg. Clin. North Am. vol. 17, pp. 349-360, 2009.

[6]H. H. El-Marakby, "The versatile naso-labial flaps in facial reconstruction,” J Egypt Natl Canc Inst. vol. 17, pp. 245-250, 2005. 
[7]W. M. Weathers, E. M. Wolfswinkel, H. Nguyen, and J. F. Thornton, "Expanded uses for the nasolabial flap," in Seminars in plastic surgery, 2013, vol. 27,pp. 104.

[8]B. Hynes and J. B. Boyd, "The nasolabial flap: axial or random?," Arch. Otolaryngol. Neck Surg., vol. 114, pp. 1389-1391, 1988.

[9]S. L. Spear, S. S. Kroll, and S. Romm, "A new twist to the nasolabial flap for reconstruction of lateral alar defects.," Plast. Reconstr. Surg., vol. 79, pp. 915-920, 1987.

[10]Ö. Karataş Silistreli, C. Demirdöver, M. Ayhan, Y. Öztan, M. Görgü, and B. G. Ulusal, "Prefabricated Nasolabial Flap for Reconstruction of Full-Thickness Distal Nasal Defects," Dermatologic Surg., vol. 31, pp. 546-552, 2005.

[11]J. A. Hassan, "Reconstruction of the Nasal Defects by Nasolabial Flaps," J. US-China Med. Sci., vol. 13, pp. 64-79, 2016.
[12]M. Javaid, A. Ahmad, Q. Khan, F. Wahid, and H. R. Afridi, "Outcome of nasolabial flap in reconstruction of the nasal alar defect," J Med Sci, vol. 19, pp. 163-166, 2011.

[13]K. Irfanullah, "A., Khan, N., Shah, SA and Tahmeedullah (2012) Indications and Outcome of Nasolabial Flap: Experience at a Plastic Surgical Facility," Ann. Pakistan Inst. Med. Sci.vol. 8, pp. 168171.

[14]M. Bilal, "Nasolabial flap: a workhorse for the reconstruction of nasal ala after tumor resection, J. Khyber Coll," Dent, vol. 5, pp. 10-15, 2014.

[15]K. S. Massoud, "Reconstruction of full-thickness alar defect by the turnover nasolabial flap: improving the outcome by primary flap-thinning and unilateral alar base suturing," Egypt J. Plast. Reconstrictive Surg., vol. 33, pp. 15-19, 2009. 